\title{
Providing Useful Data Reliably to Mobile Cloud Users from Random Wireless Sensor Network
}

\author{
Shanthosh Palani
}

MCA, School of Information Technology and Engineering, VIT University, Vellore, Tamilnadu, 632014,India.

\begin{abstract}
The integration of mobile cloud computing and wireless sensor network plays an important role in the field of research and industry. Data fetched from Wireless Sensor Network (WSN) are stored in the cloud and provides it to the mobile cloud users. This integration helps in providing data to users in the form of digitalization. To help the applications involving an integration of Mobile Cloud Computing (MCC)-Wireless Sensor Network, this paper finds the crucial things that influence the sensor data and WSN, and then propose a concept called Time based Priority Sleep Scheduling (TPSS). TPSS is divided into three parts 1 . Based on the priority and time, wireless sensor network gateway should transmit the sensed data to cloud and 2 . The algorithm used to save the battery consumption of mobile users in fetching data from the cloud is Priority Based Sleep Scheduling (PBSS); this provides data to users request based on the time slot. 3. Location Based Sleep Scheduling (LBSS) algorithm used to provide sensed data based on mobile cloud users location.
\end{abstract}

Index Terms: Wireless Sensor Network, Mobile Cloud Computing, Integration, Time based Priority Sleep Scheduling, Priority Based Sleep Scheduling, Location Based Sleep Scheduling.

(C) 2017 Published by MECS Publisher. Selection and/or peer review under responsibility of the Research Association of Modern Education and Computer Science

\section{Introduction}

Information gathering ability of wireless sensor networks (WSN) and additionally the information stockpiling and preparing capacity of mobile cloud computing (MCC), WSN-MCC combination is pulling in critical consideration from both the scholarly world and industry. Focusing on preparing of the sensory information in MCC-WSN integration, by finding the crucial issues concerning MCC-WSN integration and proposing a sensory information processing framework (in fig.2). This aims at transmitting required sensory information to the mobile users in a quick, reliable, furthermore and secure way.

* Corresponding author. Tel.:

E-mail address: shanthosh.vit@gmail.com 


\section{A. Mobile Cloud Computing}

Smart mobile phone, cloud computing and tablets come together in the latest and quickly emergent in the field of mobile cloud. Mobile cloud is the grouping of the wireless network, mobile computing and cloud computing to transport wealthy computational resources to network providers, cloud providers, and mobile users [1]. Data stored in the cloud platform are accessed by the wireless connection. Mobile client can ask for information from the relevant application provided to them. The request is then sent to the cloud and the result is shown in the application through the mobile cloud.

The main theme of the present work is to combine both wireless sensor networks and mobile cloud computing, which have to consistently provide data to mobile cloud users by Time based priority Sleep Scheduling - TPSS and Location Based Sleep Scheduling - LBSS.

\section{B. Wireless Sensor Network}

The type of architecture used in wireless sensor network is a hybrid one. It is a grouping of infrastructure less and infrastructure oriented network. Wireless sensor network consists of its own independent sensor to monitor several kinds of environmental changes and some sort of physical changes for example air, humidity, the wind, temperature, soil, pressure etc [1-3]. Wireless sensor network is made up of nodes where each node is connected to one of its sensors to fetch the details [2]. The best illustration is a forest fire, the pre-installed sensors in the forest will monitor the climate condition, temperature, gas, and humidity these helps in detecting the forest fire in an appropriate method and without the help of human manual inspection the range of wire will be determined [4] (in fig.1).
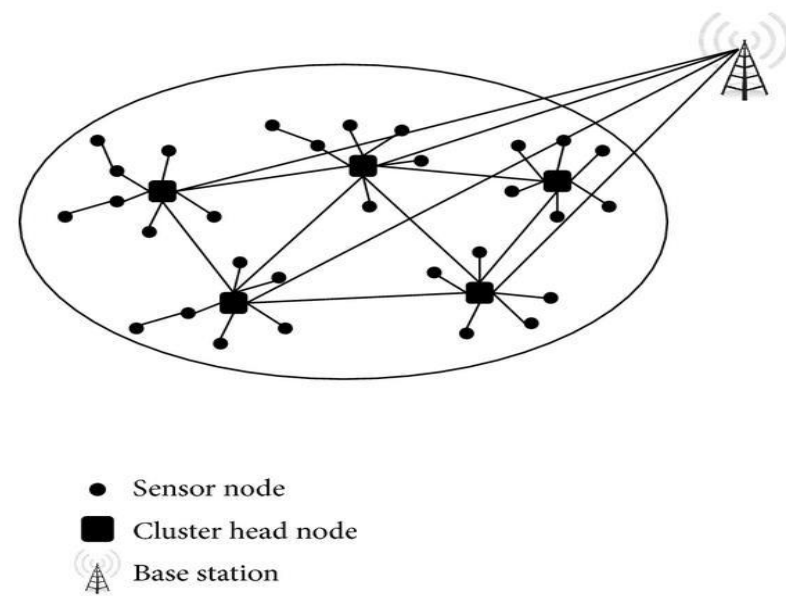

Fig.1. Wireless Sensor Network 


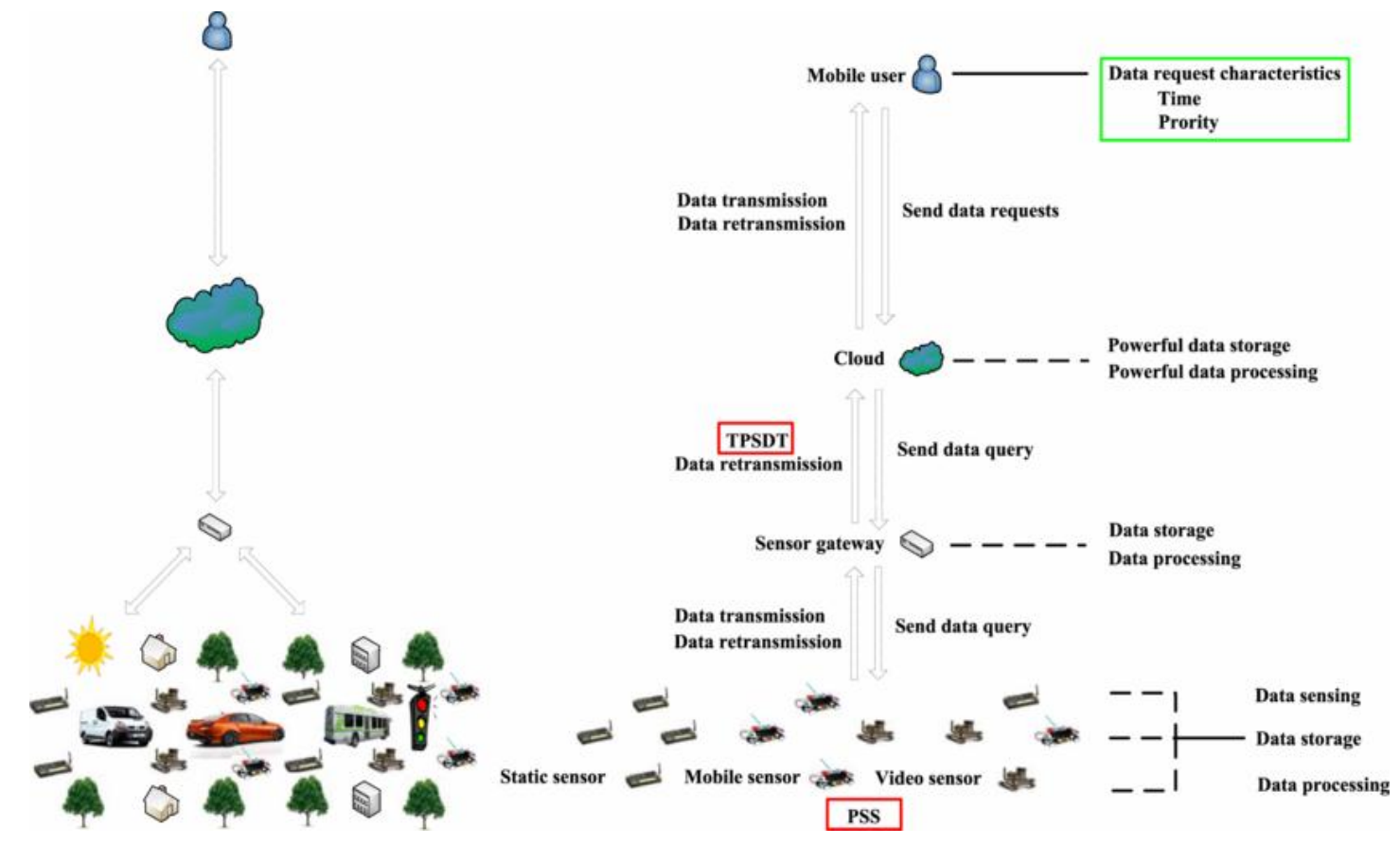

Fig.2. MCC-WSN Integration Framework

\section{Related Works}

This section discusses the state of the art research being done in this field.

- Sukanya C.M, Priya K.V, Vince Paul and Mr.Sankaranarayanan P.N [5], proposed the method that helps in integrating both wireless sensor network and mobile cloud computing. The other problem in mobile sensor networks like data supervision and communication is specified. Many researchers propose integrating WSN and MCC to provide sensed data to mobile cloud users reliably. Here authors proposed a new concept that decreases the memory storage necessity for network gateway and sensor nodes. It also minimizes the bandwidth requirement and traffic overload for wireless sensor network. Integration of WSN and MCC is done with the help of cloud. Security of the sensed data will be obtained. The requested data will be provided to mobile cloud users.

- Y.Padma, K.Pavan Kumar and A.Durgabhavani [6], they examined that an increase in the use of mobile cloud computing concepts has rapidly become higher in the recent year. It also assists in finding the distance between the cloud and sensor network and to predict the time required to communicate with each other. Mobile cloud computing influence in finding nearby and far away clouds to boost its speed in fetching data and improve the performance of mobile devices to provide reliable outputs to mobile users. The requested data can also be provided in the form of mobile application. This analysis makes clear about the disadvantages of mobile cloud and helps in finding the solutions to the problem. One of the biggest issues in the mobile cloud is battery consumption called energy and the time taken to fetch the sensed data. Mobile Cloud Computing is the combination of networking, mobile computing, and cloud computing. The type of services used in developing such mobile applications is preferably java.

- Pelin Angin, Bharat Bhargava and Zhongjun Jin [7], explained how we attain difficult jobs in real time we use mobile cloud computing in latest years with the limited usage of battery and power. Mobile cloud computing is used to overcome the drawbacks of mobile devices for recent years. Mobile cloud 
computing is capable of defeating the restrictions of mobile devices for valid computing, the techniques and technologies used for program relocation made it tough to take up mobile cloud computing due to lack of its structure. The carrying out time in the cloud of mobile application availability is basically relying on the availability of cloud. The offloading computation technique used in this module based on the independent agents. This approach does not need any rations on cloud stage except offering remote execution space and improves the supervision load of offloaded rules by mobile users using perfect, independent application division. Mobile cloud computing performance has been investigated based on the runtime of the cloud environment and provides an easy overhead vibrant integration model into independent representative to improve with self-evaluation based on the performance with selfintegration capability. To provide best possible results the offloading technique is used with the mobile cloud.

- Abid Shahzad and Mureed Hussain [8], Three service models of cloud computing have been clearly explained with relevant scenarios. Each and every cloud deployment model with their benefits was given here. There are several challenges and problems in mobile cloud computing. Some of the problems are privacy and data safety issues, in case of public data it can be easily theft, customers data privacy, failure of physical protection, the way of management in keys, Data reliability, Data should be kept safe, access rights should be given based on the user, offloading, battery usage is the main challenge in cloud computing, processing time, Bandwidth based space allocation. Now a day's several fields started to use cloud computing techniques as it is easy to maintain and scalable resource. Mobile cloud computing is a new trend to the digitalizing world. MCC allows mobile users to access cloud services from their mobile devices. MCC reduces the concert, compatible and need of source issue in cloud atmosphere.

\section{Scope and objective}

The main scope of the present work is to integrate both Wireless Sensor Networks and Mobile Cloud Computing, which will be consistently providing the data from cloud to mobile users with the help of two algorithms Time based priority Sleep Scheduling and Location Based tracking Sleep Scheduling.

Smart mobile phone, cloud computing and tablets come together in the latest and quickly emergent in the field of mobile cloud. Mobile cloud is the grouping of wireless sensor network, mobile and cloud computing to transport wealthy computational resources to network providers, cloud providers and mobile users. Data stored in the cloud platform are accessed by the wireless connection [5]. Mobile client can ask for the information from the relevant application provided to them. The request is then sent to the cloud and the result is shown in the application through the mobile cloud.

Because of the following operations performed by the sensor the power of the battery will be reduced, those operations are data transmission, processing, and sensing [8]. While performing these operations continuously the battery will get down gradually. The batteries attached with sensors will not be replaced or recharged. Since it is difficult to maintain the battery performance it is said that there must me some different method to obtain the values from the sensor. Sensors nearer to the gateway will sense a number of values and sends back to the gateway frequently [8]. So the sensors which are all nearer to the gateway will be the main reason for battery draining problem. These will lead in forming a gap in the sensor network and data will not be gathered for the cloud. Wireless network will be disconnected because of the gap in the network.

The transfer of data from one sensor to another sensor will cause crash or loss in data. The data from the wireless network to the cloud will also lead loss in data due to several issues such as low bandwidth, network overcrowding or obstruction [8]. In this kind of cases, if Wireless Sensor Network cannot achieve data retrieval, the data will not be received by cloud from wireless network.

If there is any lack in storing the sensed values in the case of low availability of memory space, then the data from the sensor will not be gathered by cloud, even though there is a sufficient power in the sensor battery to collect and pass the data from the wireless network to the cloud. 


\section{Features}

The integration of mobile cloud computing with wireless sensor network helps in processing the fetched data from wireless sensor network by cloud computing and to provide the data with the mobile user. The user preferences were extracted and the required data are rendered user mobile device automatically. Each wireless sensor network connected to the gateway sends the sensed information to the gateway [15]. Whenever the user requires the information sensed by the network, the mobile user sends the request to the cloud. The cloud stores the information sensed by the gateway. Depending on the user's request, Time-based priority sleep scheduling table will be maintained. Then the cloud sends the priority table to the each gateway. Now the gateway sends the request to the particular sensor and gets the sensory data based on the priority table. A sensor which collects the highest priority data is active then all the other sensors are in sleep state.

In this work, RACE architecture is a life cycle of the implementation. It will provide the various security aspects using incentive protocol. This protocol works between the source and destination node. Every node temporarily stores the reports and evidence and submits it to Trusted Party to get the payment correctly [9]. In an enhancement, LPSS is implemented. Based on the individual user's request, LPSS user table will be maintained. Now gateway sends the request to the particular sensor and gets the sensory data based on the user history. A sensor which it collects the user data based on LPSS are in active then all the other sensor are in the sleep state.

\section{Problem Definition}

- The data fetched by sensors need a set of energy to communicate with each other.

- Sensor network issues like message passing and data organization.

- Need higher bandwidth and traffic in the cloud increases of the sensor network.

- Users are given with on claim information or will provide all the data.

\section{Problem Formulation}

Due to the following operations performed by the sensor the power of the battery will be reduced, those operations are data transmission, processing, and sensing. While performing these operations continuously the battery will get down gradually. The batteries attached with sensors will not be replaced or recharged. Since it is difficult to maintain the battery performance it is said that there must me some different method to obtain the values from the sensor. Sensors nearer to the gateway will sense a number of values and sends back to the gateway frequently [9]. So the sensors which are all nearer to the gateway will be the main reason for battery draining problem. These will lead in forming a gap in the sensor network and data will not be gathered for the cloud. Wireless network will be disconnected because of the gap in the network.

The transfer of data from one sensor to another sensor will cause crash or loss in data. The data from the wireless network to the cloud will also lead loss in data due to several issues such as low bandwidth, network overcrowding or obstruction. In this kind of cases, if wireless sensor network cannot achieve data retrieval, the data will not be received by cloud from the wireless network.

If there is any lack in storing the sensed values in the case of low availability of memory space, then the data from the sensor will not be gathered by cloud, even though there is sufficient power in the sensor battery to collect and pass the data from the wireless network to cloud.

\section{Simulation Methodology}

The integration of mobile cloud computing with wireless sensor network helps in processing the fetched data from wireless sensor network by cloud computing and to share the requested data with the mobile user. The 
user preferences were extracted and the required data's are provided to mobile user device automatically. Each wireless sensor network connected to the gateway sends the sensed information to the gateway. Whenever the user requires the information sensed by the network, the mobile user sends the request to the cloud. The cloud stores the information sensed by the gateway [10]. Depending on the user's request, Time-based priority sleep scheduling table will be maintained. Then the cloud sends the priority table to the each gateway. Now gateway sends the request to the particular sensor and gets the sensory data based on the priority table. A sensor which collects the highest priority data is in active then all the other sensors are in sleep state. Location Based Sleep Scheduling algorithm is used to send the information based on their request and with the help of users current location.

\subsection{Considered Network Model}

We have to create multiple wireless sensor network networks. Each sensor senses the unique data such as temperature, air, co2, soil, pressure and wind etc. Each wireless sensor network has its own gateway. Each wireless sensor network Sensor is listed in a gateway. The sensors sense only the data's offered by the gateway depending upon the MCC user preference [10]. The assumption made here is that the sensors have enough memory space to overcome the problem of data dropping.

\subsection{Gateway and the Mobile Cloud}

In this component, the gateway receives the information fetched by the Wireless Sensor Networks. The sensor information is stored in the gateway and then forwarded to the cloud. Multiple mobile users are created. Based on the mobile user's request, time-based priority scheduling is maintained. Each user request for data in a time period is consequently received by the gateway [10]. The data requested are stored in MCC and the priority table is maintained for every user based on the time-space. The priority table maintains user preferences for every one-minute epoch like temperature, humidity etc.

\subsection{Time-Based Priority Sleep Scheduling}

TPSS scheme is to integrate both Wireless Sensor Networks and Mobile Cloud Computing, which will be consistently providing the data from cloud to mobile users. The process of Time Based Priority Scheduling for every wireless network is to collect and pass information with respect to cloud data for each mobile user [14]. PSS helps in finding out the wakeful and sleeping status of sensors. The sensors will be put on wake state to sense a particular set of data's as offered by the gateway. The gateway obtains the current priority table from MCC to extracts the preference of the users. Based on that the appropriate WSN sensors are chosen in particular clusters and put into wake state for sensing and forwarding the data to the gateway. This ensures the energy efficiency of WSN and minimizes the network usage and congestion [7]. Each sensor senses the information based on the time occurrence and stores in the cloud for further processing. Based on the priority user gets the data using the timeslot [13].

\subsection{Location based Sleep Scheduling}

LPSS - Location Based Sleep Scheduling is implemented. Based on the individual user's location history, LPSS user location history table will be maintained. Now gateway sends the request to the particular sensor and gets the sensory data based on the user history. A sensor which it collects the user history data based on LPSS are in active then all the other sensor are in the sleep state [14]. The mobile end users are pushed with the required useful data's automatically to MCC and WSN Integration through TPSS and LPSS. 


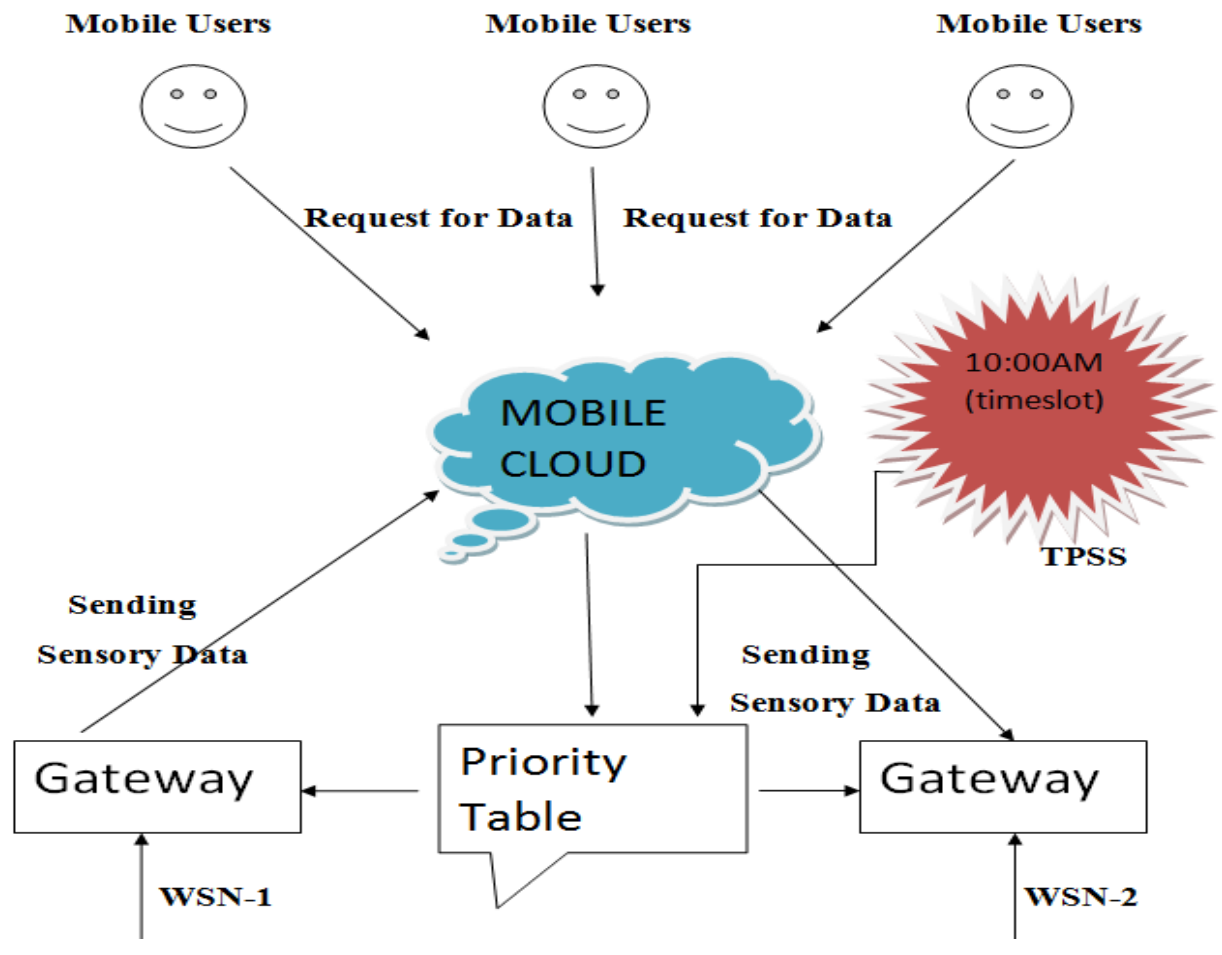

Fig.3. System Architecture

\section{Algorithm}

The WSN-MCC integration system is modelled and analyzed here based on the following assumptions.

- $\quad$ There is one cloud $\mathrm{C}$ and $\mathrm{M}$ mobile users (i.e., $\mathrm{U}=(\mathrm{u} 1, \mathrm{u} 2, . ., \mathrm{uM}))$ as well as M. WSN (i.e., WSN $=($ wsn1, wsn2, . ., wsn M)).

- Each WSN gathers and transmits data to the cloud to satisfy the data requests from each corresponding mobile user.

- $\quad$ Each WSN consists of one gateway g as well as $\mathrm{N}$ sensor nodes (i.e., $\mathrm{I}=(\mathrm{i} 1$, i2 ......... iN)).

- Each gateway g is externally powered with an unlimited energy supply.

- Each sensor node i has a limited energy supply powered by a non-rechargeable and non-replaceable battery, which has an initial energy eo and a residual energy ei.

- $\quad$ Time is divided into $\mathrm{Z}$ time periods (i.e., $\mathrm{T}=(\mathrm{t} 1, \mathrm{t} 2, \ldots, \mathrm{tZ}))$

Assume the number of data requests issued for a point of interest (e.g., sensor node i) during each specific time period $t$ in the history is rit. In addition, given that the number of data requests issued to all points for each specific time period $t$ in the history is Rt. The probability (i.e., pti) that the data requests concern the sensor node $\mathrm{i}$ in each specific time period $\mathrm{t}$ is calculated as follow.

$$
p_{i}^{t}=\frac{r_{i}^{t}}{R^{t}}
$$


In addition, for the whole WSN-MCC integration, there is $Z$ time periods and $N$ sensor nodes. Thus,

$$
1=\sum_{\mathrm{i}=\mathrm{i} 1}^{\mathrm{i}=\mathrm{iN}} \mathrm{p}_{\mathrm{i}}^{\mathrm{t}}(\mathrm{t}=\mathrm{t} 1, \mathrm{t} 2, \ldots, \mathrm{tz})
$$

This PTP table obtained for each mobile user is updated dynamically by the cloud $C$ and sent to the gateway $g$ of each corresponding WSN.

Table.1. Example of time and priority (PTP) vs. point table.

\begin{tabular}{llllll}
\hline Point of Interest & T1 & T2 & T3 & T4 & $\ldots \ldots$. \\
\hline i1 & $11 \%$ & $6 \%$ & $21 \%$ & $16 \%$ & $\ldots \ldots$. \\
i2 & $21 \%$ & $6 \%$ & $0 \%$ & $16 \%$ & $\ldots \ldots$. \\
i3 & $21 \%$ & $11 \%$ & $0 \%$ & $16 \%$ & $\ldots \ldots$. \\
i4 & $11 \%$ & $11 \%$ & $0 \%$ & $11 \%$ & $\ldots \ldots$. \\
i5 & $21 \%$ & $21 \%$ & $0 \%$ & $16 \%$ & $\ldots \ldots$. \\
$\ldots \ldots$ & $\ldots \ldots$. & $\ldots \ldots$ & $\ldots \ldots$ & $\ldots \ldots$ & $\ldots \ldots$ \\
\hline T1- 9am - 10am, T2-10am - 11am, T3 11am - 12pm, T4 12pm-1pm
\end{tabular}

\subsection{Pseudo code of PSS Algorithm}

First: Run the following at gateway g during each time period $t$.

Step 1: Gateway g obtains PTP table.

Step 2: If pti $>0$, g sends flag A to node i.

Step 3: Run the second part at each node i.

Second: Run the following at each node i during each time period t.

Step 1: Get the current residual energy rank ei.

Step 2: Broadcast ei and receive the energy ranks of its currently awake neighbours Ni. Let Ei be the set of these ranks.

Step 3: Broadcast Ei and receive $\mathrm{Ej}$ from each $\mathrm{j} \in \mathrm{Ni}$.

Step 4: If $|\mathrm{Ni}|<\mathrm{k}$ or $|\mathrm{Nj}|<\mathrm{k}$ for any $\mathrm{j} \in \mathrm{Ni}$, remain awake.

Go to Step 7.

Step 5: Compute $\mathrm{Ci}=\{\mathrm{j} \mid \mathrm{j} \in \mathrm{Ni}$ and $\mathrm{ej}>\mathrm{ei}\}$.

Step 6: Go to sleep if both the following conditions hold.

Remain awake otherwise.

- Any two nodes in Ci have connected either directly themselves or indirectly through nodes within i's 2-hop neighbourhood that have e more than ei.

- Any node in Ni has at least $\mathrm{k}$ neighbours from $\mathrm{Ci}$.

- It does not receive flag A.

Step 7: Return.

\subsection{MCC - WSN Integration method}

The detailed steps of TPSS for each WSN to gather and transmit sensory data for each corresponding mobile user are depicted as follows. 
- Sensor nodes determine their awake and asleep states with PSS.

- Sensor nodes sense the environmental data with a set frequency and store the sensory data as well as process the sensory data.

- Sensor nodes send the processed sensory data to the gateway g with the many to one and hop by hop pattern.

- Gateway g stores the received sensory data and then processes the sensory data.

- Gateway g selectively transmits the sensory data to the cloud C with TPSDT.

- Cloud $\mathrm{C}$ further stores and processes the received sensory data.

- If data transmission from $\mathrm{i}$ to $\mathrm{g}$ or $\mathrm{g}$ to $\mathrm{C}$ experiences data losses or failures, $\mathrm{i}$ or $\mathrm{g}$ performs data retransmission until the data transmission is successful.

- Mobile user $\mathrm{u}$ issues data requests to cloud $\mathrm{C}$ and cloud $\mathrm{C}$ transmits the requested sensory data to the mobile user $u$.

- If data transmission from $\mathrm{C}$ to $\mathrm{u}$ encounters data losses or failures, $\mathrm{C}$ performs data retransmission until the data transmission is successful.

- Cloud C dynamically updates the PTP table with equation (1) if the time and priority features of the requested data of the mobile user are changed and sends the updated PTP table to the gateway in each time period $\mathrm{t}$.

\section{Result}

The evaluation results with respect to the usefulness of sensory data and reliability of WSN for each mobile user in time basis are determined.

We have observed that, averaging over all mobile users, around $85 \%$ of the sensory data sent to the cloud with TPSS are useful to the mobile users, whereas only around $45 \%$ of the sensory data sent to the cloud with GS are useful to the mobile users. The same results are obtained for each of the four weeks observed. This demonstrates that TPSS greatly improves the usefulness of sensory data due to the fact that mobile users generally request data over time according to the PTP tables.

Also, we can observe that the reliability of WSN is also greatly enhanced with TPSS comparing GS. Particularly, the three sub-figures show that over each of the four weeks observed, the reliability of WSN with GS is around 180 hours for all the mobile users, while the reliability of WSN with TPSS varies among different mobile users but averages to around 400 hours.

In summary, TPSS substantially outperforms GS in terms of usefulness of sensory data and reliability of WSN. Moreover, for different mobile users with various data request characteristics indicated by different PTP tables, the usefulness of sensory data varies considerably for both TPSS and GS, while only the reliability of WSN for TPSS changes with mobile users.

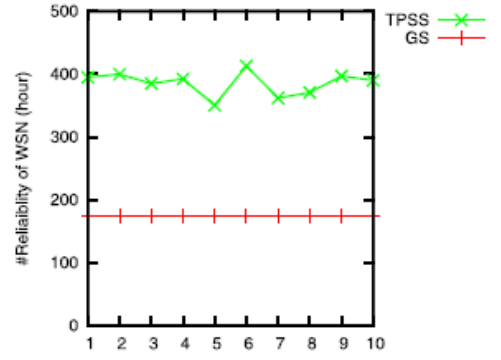

(a) In time $\mathrm{T} 1$

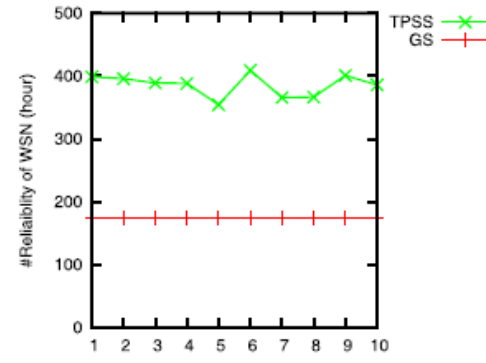

(b) In time T2

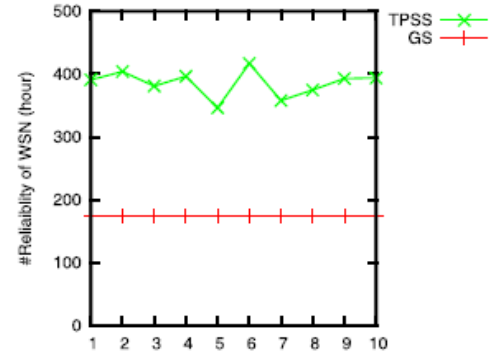

(c) In time T3

Fig.4. Average level reliability of Wireless Sensor Network 
There are a number of works related to MCC-WSN integration. They mainly focus on the following two aspects:

1) Improving the performance of WSN.

2) Better utilizing the data collected by the WSN

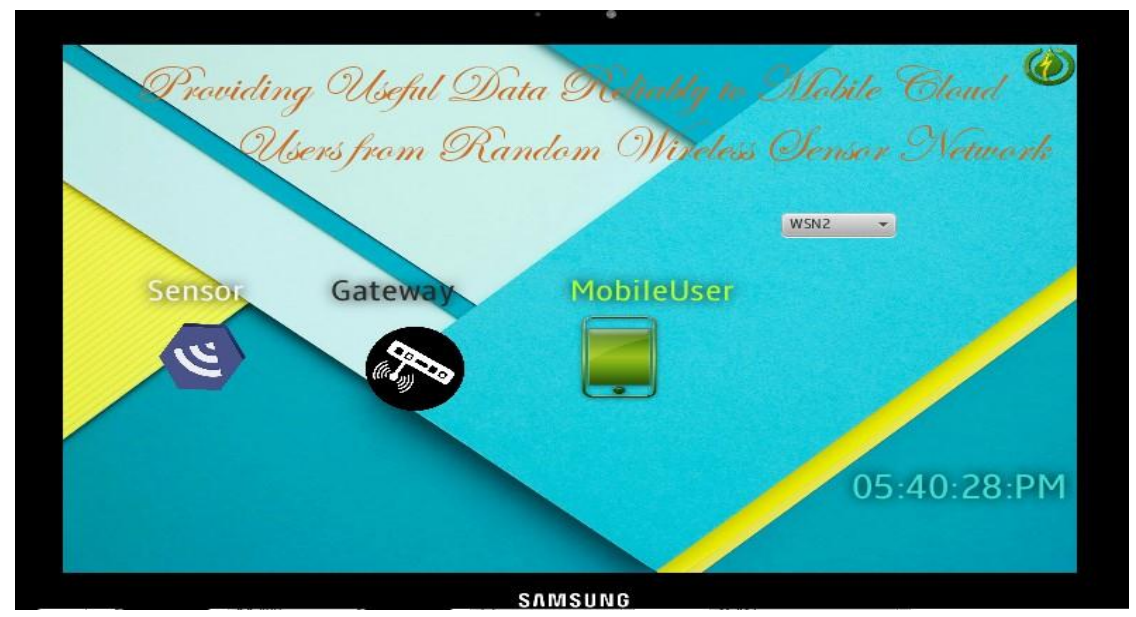

Fig.5. MCC -WSN Integration Module

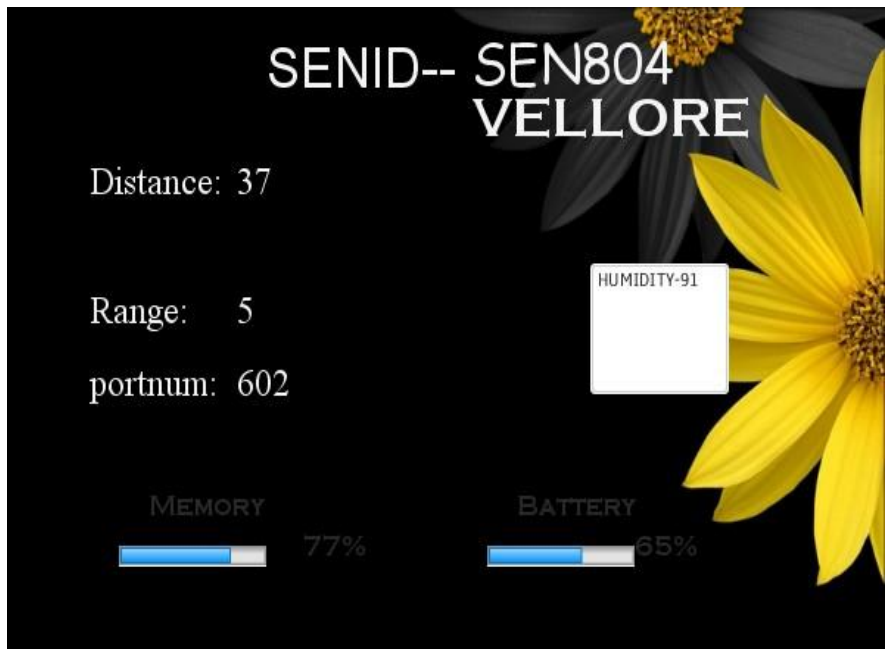

Fig 6. Sensor 


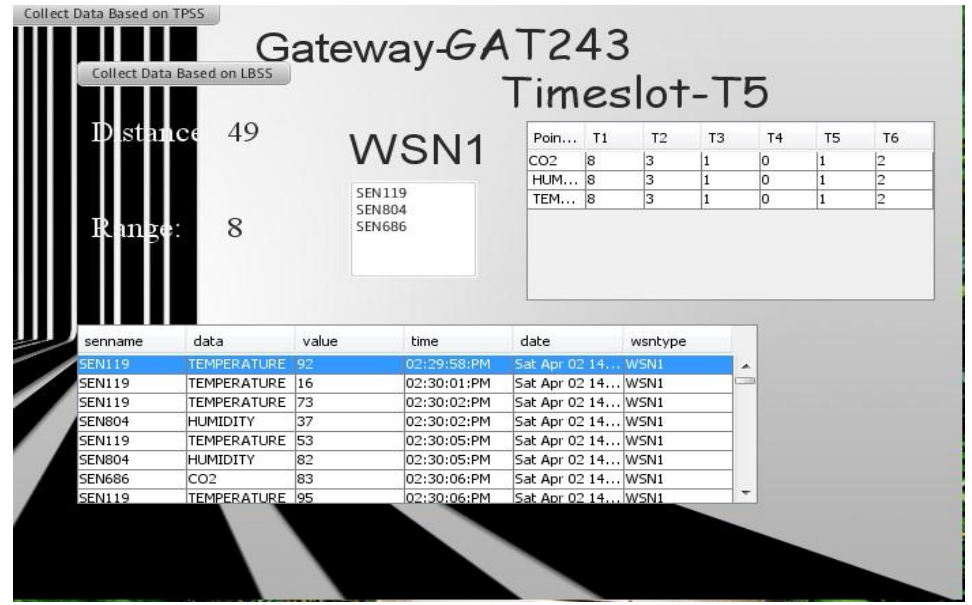

Fig.7. Gateway

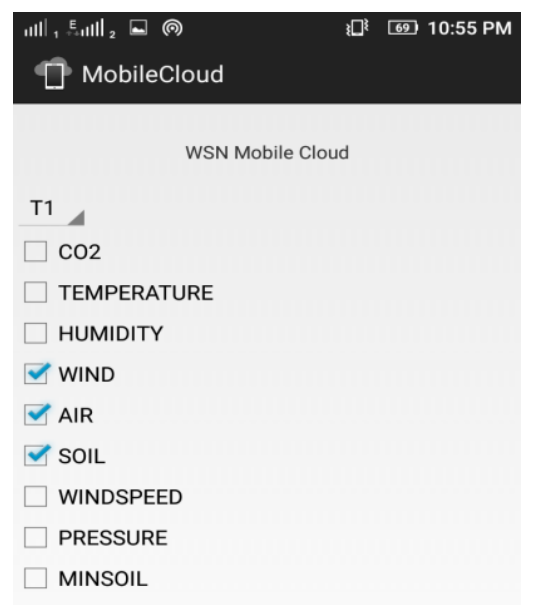

Check Server

Fig.8. Mobile cloud application 


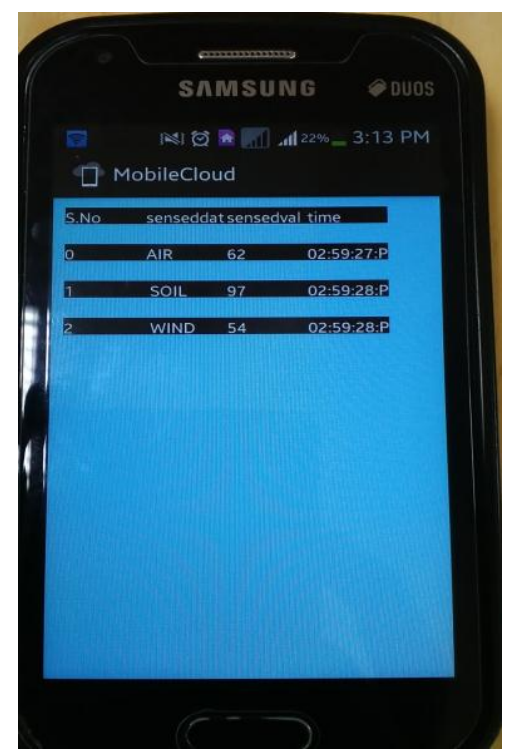

Fig.9. Mobile application result

\section{Conclusions}

In the above-entitled paper, the integration of ever present integration of WSN-MCC powerful data processes and powerful data storage potential of mobile cloud computing. It supports WSN and MCC integrated application that reliably deliver data to mobile cloud users through the application from the wireless sensor network to the cloud. In this area, we have found some issues in using the sensor data with some battery consuming process and proposed a new concept called Time based Priority Sleep Scheduling to overcome the problem. This helps in keeping the unrequested sensor to the sleep state, which helps in saving the battery power. This algorithm will consistently fetch data from the sensor and transmit to the gateway for further proceedings in the cloud. Based on the user priority it will transmit the data asked for by the mobile user. PBSS algorithm helps in providing the sensor data depending upon the time priority, this helps to put the unwanted sensor in the sleep state. In this paper, two methods are proposed to resolve the drawbacks of Wireless Sensor Network and Mobile Cloud Computing. Those methods are TPSS and LPSS. Based on the time the information furnished to users with the help of TPSS algorithm and based upon the location of the user LBSS algorithm will be helping the cloud to furnish the information. This two algorithm uses wakeful and sleeps status to save the battery performance. Results have proven that these methods will be suitable for integrating wireless sensor network and mobile cloud computing is effective.

\section{Acknowledgements}

I would like to express my special gratitude and thanks to my guide Prof Mary Mekala A, Assistant Professor Senior, School of Information Technology and Engineering, VIT University, whose esteemed guidance and immense support encouraged to complete the research work successfully. I thank the Management of VIT University for permitting me to use the library resources. I also thank all the faculty members of VIT University for giving me the courage and strength I needed to complete my goals. This acknowledgement would be incomplete without expressing my whole hearted thanks to my family and friends who motivated me during the course of the work. 


\section{References}

[1] Gursimran Kaur and Geetanjali Babbar, "A Novel RTS/CTS based Relative Time Synchronization Technique for RFID based Wireless Sensor Network" IJWMT - International Journal of Wireless and Microwave Technologies, 2015, 5, 35-46

[2] Ibrahim S. I. Abuhaiba, Amina Y. Al-Sallut, "Intelligent and Distributed Localization of Nodes in Wireless Sensor Networks" IJWMT - International Journal of Wireless and Microwave Technologies, 2015, 2, 22-36.

[3] C. Zhu, V. C. M. Leung, X. Hu, L. Shu, and L. T. Yang, “A review of key issues that concern the feasibility of mobile cloud computing," in Proc. IEEE Int. Conf., Cyber, Phys. Soc. Comput. (CPSCom), Aug. 2013,pp. 769_776.

[4] C. Zhu, L. Shu, T. Hara, L. Wang, S. Nishio, and L. T. Yang, “A survey on communication and data management issues in mobile sensor networks," Wireless Commun. Mobile Computing vol. 14, no. 1, pp. 19_36, Jan. 2014.

[5] Sukanya C.M, Priya K.V, Vince Paul and Mr.Sankaranarayanan P.N, "Integration of Wireless Sensor Networks and Mobile Cloud- a Survey", IJCSIT - International Journal of Computer Science and Information Technologies, Vol. 6 (1), 2015, 159-163.

[6] Y.Padma, K.Pavan Kumar and A.Durgabhavani, "A Simple Analysis of Service Oriented Architecture for Mobile Cloud Computing", International Journal of Engineering Trends and Technology (IJETT) Volume 11 Number 1 - May 2014.

[7] Pelin Angin, Bharat Bhargava and Zhongjun Jin, "A Self-Cloning Agents Based Model for High Performance Mobile-Cloud Computing", 2015 IEEE 8th International Conference on Cloud Computing Volume June 27 2015-July 22015.

[8] Abid Shahzad and Mureed Hussain, "Security Issues and Challenges of Mobile Cloud Computing". International Journal of Grid and Distributed Computing Vol.6, No.6 (2013), pp. 37-50.

[9] S. Abolfazli, Z. Sanaei, M. Alizadeh, A. Gani, and F. Xia," An experimental analysis on cloud-based mobile augmentation in MCC," IEEE Trans.Consum.Electron., Feb. 2014.

[10] Chunsheng Zhu, Zhengguo Sheng, Victor C. M. Leung, Lei Shu and Laurence T. Yang, "Toward offering more useful data reliably to mobile cloud from wireless sensor network", IEEE Transactions on Emerging Topics in Computing, March 2015.

[11] M. Li and Y. Liu, "Underground coal mine monitoring with wireless sensor networks," ACM Trans. Sensor Netw., vol. 5, no. 2, Mar. 2013, Art. ID 10.

[12] I. F. Akyildiz, W. Su, Y. Sankarasubramaniam, and E. Cayirci, "Wireless sensor networks: A survey," Computer Network., vol. 38, no. 4, pp. 393_422, Mar. 2012.

[13] Pelin Angin, Bharat Bhargava and Zhongjun Jin, "A Self-Cloning Agent-Based Model for HighPerformance Mobile-Cloud Computing". IEEE 8th International Conference on Cloud Computing 2015.

[14] C. Zhu, V. C. M. Leung, L. T. Yang and L. Shu, Collaborative location based sleep scheduling for wireless sensor networks integrated with mobile cloud computing' IEEE Trans. Comput., vol. PP, no. 99, p.1, published Aug. 2014.

[15] S. Misra, S. Bera, A. Mondal, R. Tirkey, H.-C. Chao, and S. Chattopadhyay, "Optimal gateway selection in sensor-cloud framework for health monitoring," IET Wireless Sensor Syst., vol. 4, no. 2, pp. 61-68, Jun. 2014. 


\section{Authors' Profiles}

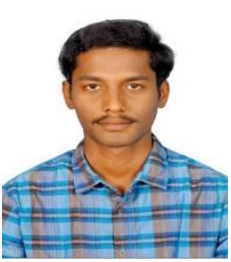

Shanthosh Palani has completed his Master's in Computer Applications from VIT University, Vellore, Tamilnadu, India in 2016. He has done his Bachelor's in Computer Application from VIT University, Vellore, Tamilnadu, India in 2014. Presently he is working at Tata Consultancy Services Limited, Chennai, Tamilnadu, India as Assistant Systems Engineer at Data Center. His research interest's lies in networks, wireless communication and cloud computing.

How to cite this paper: Shanthosh Palani,"Providing Useful Data Reliably to Mobile Cloud Users from Random Wireless Sensor Network", International Journal of Wireless and Microwave Technologies(IJWMT), Vol.7, No.1, pp.49-62, 2017.DOI: 10.5815/ijwmt.2017.01.06 